\title{
Drugs for Lupus While Breastfeeding
}

\author{
Philip O. Anderson
}

$\mathbf{S}$ YSTEMIC LUPUS ERYTHEMATOSUS (SLE) is most prevalent in women during their reproductive years. Thus, the issue of drug treatment of the disease during breastfeeding is not uncommon. Meta-analyses show that prolactin appears to exacerbate SLE, and that prolactin levels are correlated with disease activity. ${ }^{1,2}$ Bromocriptine, which lowers serum prolactin, appears to decrease disease activity in postpartum women with SLE. $^{3}$ One group of investigators regards breastfeeding to be contraindicated in patients with SLE because breastfeeding results in increased serum prolactin. ${ }^{4}$ Whether breastfeeding exacerbates SLE that is well controlled by drug therapy is not clear and international treatment guidelines do not proscribe breastfeeding in women with SLE.

As in other diseases, mothers with SLE may discontinue breastfeeding because of taking medications, although the discontinuation rate seems to be higher in this group of women. A cohort study from Duke University found that $54 \%$ of women with SLE who had stopped breastfeeding by 6 weeks postpartum did so because of taking a medication. ${ }^{5}$ A casecontrol study from Argentina found that mothers with SLE failed to initiate breastfeeding at a higher rate than control mothers. When they did breastfeed, they stopped sooner and $41 \%$ of mothers weaned because of receiving a medication, although the medications they received were compatible with breastfeeding. ${ }^{6}$

A wide variety of immunosuppressant medications are used to treat SLE. The various drug classes are reviewed in this column. The therapeutic scheme for drug choice in SLE is primarily based on European League Against Rheumatism (EULAR) guidelines. ${ }^{7}$ Additional literature references on specific drugs can be found in the corresponding LactMed records.

\section{Antimalarials}

Hydroxychloroquine is a foundation of SLE treatment. A recently published study from China reported on 33 women who had been taking hydroxychloroquine sulfate for at least 1 year and were exclusively breastfeeding. They had hydroxychloroquine levels in milk determined five times during a 12-hour period just before and after a dose. The highest concentrations in milk occurred 2-4 hours after the dose. The estimated infant daily dosage averaged $0.1 \mathrm{mg} / \mathrm{kg}$ with doses of 100 or $200 \mathrm{mg} /$ day and $0.2 \mathrm{mg} / \mathrm{kg}$ with doses of $400 \mathrm{mg} / \mathrm{day}$. These corresponded to weight-adjusted infant dosages of $1.9-3.2 \%$ of the maternal dosage. Follow-up of the infants at 1 year did not find ocular toxicity or growth abnormalities.

These finding are consistent with other studies that found low levels in milk and that infants exposed to hydroxychloroquine during breastfeeding receive only small amounts of the drug. Careful follow-up found no adverse effects on growth, vision, or hearing in infants up to at least 1 year of age. Virtually all experts agree that hydroxychloroquine is acceptable during breastfeeding.

\section{Corticosteroids}

Low-dose oral corticosteroids are often used as maintenance drugs in SLE. No adverse effects have been reported in breastfed infants with maternal use of any systemic corticosteroid during breastfeeding. No waiting time is necessary before nursing after doses of oral corticosteroids, or low-dose injectable corticosteroids. Occasionally $0.25-1 \mathrm{~g}$ pulses of intravenous (IV) methylprednisolone are given daily for 1-3 days for disease flares. With maternal IV doses of methylprednisolone $1 \mathrm{~g}$, fully breastfed infants would receive doses less than their daily cortisol output, and much less than a therapeutic dose on the day of infusion. Accumulation of the drug does not occur in breast milk with three consecutive daily IV doses. Avoiding breastfeeding during the infusion and for as little as 2 hours after $1 \mathrm{~g}$ IV dose would markedly reduce infant exposure. Breastfeeding abstinence for 2-4 hours would further reduce the infant dose.

\section{Small Molecule Immunosuppressants}

Oral immunosuppressants are often added to hydroxychloroquine with or without a low-dose corticosteroid if symptoms are not controlled. Studies in women with SLE, inflammatory bowel disease, or organ transplantation taking doses of azathioprine up to $200 \mathrm{mg}$ /day for immunosuppression have found either low or unmeasurable levels of the active metabolites in milk and infant serum. Some evidence indicates a lack of adverse effects on infants exposed to azathioprine during breastfeeding up to 3.5 years of age, but long-term follow-up for effects such as carcinogenesis has not been performed. Mothers with decreased activity of the enzyme that detoxifies azathioprine metabolites may transmit higher levels of drug to their infants through breast milk, but no instances of this phenomenon have been reported. Cases of mild asymptomatic neutropenia have been reported; the

Division of Clinical Pharmacy, Skaggs School of Pharmacy and Pharmaceutical Sciences, University of California, San Diego, La Jolla, California. 
desirability of monitoring exclusively breastfed infants with a complete blood count with differential and liver function tests is debatable. Avoiding breastfeeding for 4 hours after a dose should markedly decrease the dose received by the infant in breast milk.

Mercaptopurine is both an active metabolite of azathioprine and a drug used itself for SLE. Much less information is available on the use of mercaptopurine during breastfeeding than for azathioprine. A patient receiving $50 \mathrm{mg} /$ day of mercaptopurine was recently reported. Her milk was free of the drug and its active metabolites 4 hours after the dose. Most experts consider breastfeeding during azathioprine and mercaptopurine therapy to be acceptable in nursing mothers.

Methotrexate and mycophenolate are other immunosuppressants that are used to treat SLE not adequately controlled by hydroxychloroquine and a corticosteroid. A maternal dose of methotrexate up to $92 \mathrm{mg}(1.12 \mathrm{mg} / \mathrm{kg})$ produces low levels in milk, leading some authors to state that the low doses of 2.5-25 mg/week used for SLE present a low risk to the breastfed infant. However, some expert opinion warns against this use. Withholding breastfeeding for 24 hours after a weekly low dose of methotrexate would decrease the infant's dose by $\sim 40 \%$. If breastfeeding is undertaken during long-term low-dose methotrexate use, monitoring of the infant's complete blood count and differential could be considered. Methotrexate's effects are reversible with folinic acid (leucovorin), but not with folic acid.

No information is available on the extent of mycophenolate's excretion into breast milk. The Transplantation Pregnancy Registry has collected information on six mothers who breastfed six infants while taking a mycophenolate product post-transplant. The maximum time that any of the infants was breastfed was 14 months. None of the infants had any adverse reactions reported.

Cyclosporine is used as maintenance therapy in more severe presentations of SLE. The concentration in milk is variable, but with typical maternal cyclosporine blood levels, a completely breastfed infant would usually receive no more than $\sim 2 \%$ of the mother's weight-adjusted dosage or pediatric transplantation maintenance dosage, and often $<1 \%$. Most infants studied have not had detectable cyclosporine blood levels, but two infants have had measurable cyclosporine levels, one with blood levels in the therapeutic range despite relatively low maternal milk levels. Because of extensive experience in transplant patients, most experts consider cyclosporine compatible with breastfeeding. Breastfed infants should be monitored if this drug is used during lactation, possibly including measurement of serum levels to rule out toxicity if there is a concern.

Tacrolimus is sometimes used to treat lupus nephritis. Levels of tacrolimus in milk are low and probably do not adversely affect the breastfed infant. Most experts consider tacrolimus to be probably safe to use for inflammatory bowel disease and post-transplant in nursing mothers. Exclusively breastfed infants should be monitored if this drug is used during lactation, possibly including measurement of serum levels.

Cyclophosphamide is sometimes used in SLE to treat severe organ- or life-threatening conditions as well as a rescue therapy in patients not responding to other therapies. Cyclophosphamide appears in milk in potentially toxic amounts; additionally, highly toxic active metabolites could add to the risk to the infant. In one patient, cyclophosphamide was absent from breast milk 24 hours after a single $2.8 \mathrm{~g} \mathrm{IV}$ dose, but metabolites were not measured. In another recent case, it took 7 days after a cyclophosphamide dose of $750 \mathrm{mg} / \mathrm{m}^{2}$ to fall to low levels in milk, with the drug and some metabolites still detectable in milk 3 weeks after the dose. Neutropenia has been reported in two infants whose mothers breastfed them while receiving cyclophosphamide. Breastfeeding should be stopped temporarily if nursing mothers need a short course of cyclophosphamide. A safe time to resume nursing after the last dose is not well defined. Mothers requiring long-term cyclophosphamide should not breastfeed.

\section{Biologicals}

Belimumab is a monoclonal antibody that inhibits $B$ lymphocyte stimulator, thereby inhibiting survival of B cells. It is used as add-on therapy to treat moderately severe refractory SLE. Very little information is available on its use in nursing mothers. However, it is a large protein molecule with a molecular weight of $147,000 \mathrm{Da}$. The amount in milk is likely to be very low and absorption is unlikely because it is probably destroyed in the infant's gastrointestinal tract. One woman with SLE was taking belimumab at an unstated dose for 1 year when she became pregnant. She received monthly belimumab infusions during pregnancy and continued it postpartum, starting at 2 weeks postpartum. She breastfed her infant, but the extent was not reported. No infant effects were mentioned. If belimumab is required by the mother, it is not a reason to discontinue breastfeeding, ${ }^{8}$ but it should be used with close monitoring during breastfeeding, especially while nursing a newborn or preterm infant.

Rituximab is a monoclonal antibody that targets CD20 antigen on the surface of pre-B and mature B lymphocytes, mediating B cell lysis. It is usually reserved for the most severe SLE. Like belimumab, it is a large protein molecule that theoretically should not reach the infant's systemic circulation. Information from five mothers indicates that the amount in milk is very low. Two breastfed infants apparently experienced no adverse effects during maternal use of rituximab, but long-term data are inadequate. If rituximab is required by the mother, it is not a reason to discontinue breastfeeding, ${ }^{8}$ but rituximab should be used with close monitoring during breastfeeding, especially while nursing a newborn or preterm infant.

Intravenous immune globulin G (IVIG) is sometimes used to treat hematologic manifestations of SLE, such as thrombocytopenia or autoimmune hemolytic anemia. Immunoglobulin $\mathrm{G}$ (IgG) is a normal component of breast milk. Data from two mothers indicate that IgG concentrations in milk are normal or higher and IgM levels in milk are normal or lower during IVIG therapy. The antibacterial activity of milk in these women was normal. There are no concerns with the use of IVIG during breastfeeding.

\section{Summary}

SLE is a complex autoimmune disease that theoretically might be exacerbated by breastfeeding. The most commonly used drugs to treat women with SLE, such as hydroxychloroquine, corticosteroids, and azathioprine, have good evidence of safety during breastfeeding, as do cyclosporine 
and tacrolimus. Immunosuppressants used for more advanced disease have less information, but no serious adverse effects have been reported with the exception of cyclophosphamide, which should not be used during breastfeeding.

\section{Disclosure Statement}

No competing financial interests exist.

\section{References}

1. Song GG, Lee YH. Circulating prolactin level in systemic lupus erythematosus and its correlation with disease activity: A meta-analysis. Lupus 2017;26:1260-1268.

2. Wang P, Lv TT, Guan SY, et al. Increased plasma/serum levels of prolactin in systemic lupus erythematosus: A systematic review and meta-analysis. Postgrad Med 2017;129: 126-132.

3. Qian Q, Liuqin L, Hao L, et al. The effects of bromocriptine on preventing postpartum flare in systemic lupus erythematosus patients from South China. J Immunol Res 2015;2015:316965.

4. Viera Borba V, Shoenfeld Y. Prolactin, autoimmunity, and motherhood: When should women avoid breastfeeding? Clin Rheumatol 2019;38:1263-1270.
5. Noviani M, Wasserman S, Clowse ME. Breastfeeding in mothers with systemic lupus erythematosus. Lupus 2016;25: 973-979.

6. Acevedo M, Pretini J, Micelli M, et al. Breastfeeding initiation, duration, and reasons for weaning in patients with systemic lupus erythematosus. Rheumatol Int 2017;37:1183-1186.

7. Fanouriakis A, Kostopoulou M, Alunno A, et al. 2019 update of the EULAR recommendations for the management of systemic lupus erythematosus. Ann Rheum Dis 2019; 78: 736-745.

8. Gotestam Skorpen C, Hoeltzenbein M, Tincani A, et al. The EULAR points to consider for use of antirheumatic drugs before pregnancy, and during pregnancy and lactation. Ann Rheum Dis 2016;75:795-810.

Address correspondence to: Philip O. Anderson, PharmD Division of Clinical Pharmacy

Skaggs School of Pharmacy and Pharmaceutical Sciences University of California, San Diego 9500 Gilman Drive La Jolla, CA 92093-0657

E-mail: phanderson@ucsd.edu 